\title{
Invasion of the Anterior Abdominal Wall: A Case Report of a Desmoid Tumour
}

\author{
Norton SM*, McDermott A, Sehgal R and O'Donoghue G
}

University Hospital Waterford, Waterford, Ireland

\begin{abstract}
Introduction: Desmoid fibromatosis (DF) or Desmoid tumours are rare, benign musculoaponeurotic tumours. DF have no potential for metastatic spread but are locally aggressive and spread along fascial planes, leading to extensive patient morbidity. While germline Adenomatous Polyposis Coli (APC) mutations are associated with DF, it can also occur sporadically. Debate regarding the most appropriate treatment options exists in literature.

Case summary: A 38 year old lady presented with a left hypochondrial swelling, night sweats and weight loss to the General Surgical Outpatients Department at our institution. This was noticed after the birth of her second child. No relevant family history was noted. A pre-operative ultrasound demonstrated a fusiform nodule within the left anterior abdominal transversalis oblique muscle, which had a focal hypervascular nidus. Surgical excision of the mass was performed under general anaesthetic and histologically the tumour was found to contain spindled myofibroblastic cells arranged in fascicles with perivascular lymphoid infiltrates. The diagnosis was DF with a positive margin status. Given the high risk of recurrence of this tumour type, the decision was made to undertake a further resection.

Conclusion: DF is known to be a locally aggressive benign tumour of mesenchymal origin. The benefits and disadvantages of treatment options are debated within literature. Surgery is reported to have better or similar local control rates to radiotherapy but without radiation related complications. Given that DF is known to be locally aggressive, affecting young people and with a female preponderance, we believe that surgical excision with clear margins is the most appropriate treatment of choice.
\end{abstract}

\section{Introduction}

This work has been reported in line with the SCARE criteria [1]. In 1954, Stoutin (REF) described a desmoid tumour or desmoid fibromatosis (DF) as a benign non-encapsulated lesion of mesenchymal origin which has a tendency for local spread along fascial planes [2]. DF's are rare, with only 2-4 per 1,000,000 diagnosed per year ${ }^{3}$. This tumour has a female preponderance and is more common between the ages of 12-40 [3]. Histologically it is recognised by a monoclonal proliferation of myofibroblasts. Musculoaponeurotic tissue, including the abdominal wall, extremities, head and neck is most often the site of a desmoid tumour. There is no metastatic potential for this tumour however local invasion leads to extensive morbidity and mortality in these patients due to destruction of bones, organs and soft tissue.

There is a 1000 times increased risk of developing a DF in patients who have familial adenomatous polyposis (FAP) or Gardner Syndrome. $5-10 \%$ of all desmoid tumours occur in those with pre-existing FAP [4]. The association with these condition is due to germline mutations in the adenomatous polyposis coli $(A P C)$ gene. The $A P C$ gene acts to downregulate the expression of beta-catenin. In APC gene mutations, there is accumulation of beta catenin resulting in cell proliferation and stromal expansion. In sporadic cases, $85 \%$ are due to somatic mutations in the APC gene or in CTNNB1 which codes for beta-catenin [5]. An oestrogen hypothesis is described in literature as potentially playing a role in the maintenance of the tumour [6].

Treatment options for desmoid tumours include watch and wait, surgery, radiation therapy, surgery with radiation therapy or systemic therapy (anti-oestrogen treatment). Due to the low incidence of DF's, no randomised control trial has been performed. This has resulted in a lack of consensus in the literature regarding the most appropriate treatment for DF. The Cancer care Ontario's program and sarcoma disease group recommend a watch and wait approach for suitable patients who have a stable, non-aggressive lesion which is slow growing [7]. However, in a clinical setting, given the rareness of these desmoid tumours it would be difficult to differentiate this from a malignant tumour. A malignant tumour could not be ruled out without a minimum of a biopsy would be required in these patients if this approach was to be undertaken.

Some studies have favoured surgical excision alone [8]. A risk versus benefit approach must be undertaken when considering surgery in these patients. Given the potential for aggressive local spread along fascial planes and the requirement of a negative margin status surgical excision as a treatment can be a challenge due to the risk of morbidity. Surgery with radiation therapy is another treatment option. Radiation therapy can offer local control, however the risk of potential harm following radiation complications is an important consideration, especially given that this tumour has no metastatic potential. Spear et al. [9], reported that there was a local recurrence control rate of $69 \%$ and $72 \%$ at 5 years in those who underwent surgery alone versus surgery with radiation therapy, respectively. Ballo et al. [10], reported that radiation versus surgery with radiation therapy had local control rates of $75 \%$ and $76 \%$ at 10 years, respectively. However, $17 \%$ had radiation related complications. A recent study of 211 patients treated with surgery alone reported a 5 -year recurrence free survival of $52.8 \%$. This high rate of recurrence indicates a need for adjuvant treatment [11].

Several factors have been reported to influence local control of

*Corresponding author: Norton SM, Department of Surgery, University Hospital Waterford, Waterford, Ireland, Tel: +35351848 000; E-mail: sarahnorton@rcsi.ie

Received November 24, 2016; Accepted January 24, 2017; Published January 26, 2017

Citation: Norton SM, McDermott A, Sehgal R, O'Donoghue G (2017) Invasion of the Anterior Abdominal Wall: A Case Report of a Desmoid Tumour. J Mol Biomark Diagn S2: 027. doi:10.4172/2155-9929.S2-027

Copyright: (c) 2017 Norton SM, et al. This is an open-access article distributed under the terms of the Creative Commons Attribution License, which permits unrestricted use, distribution, and reproduction in any medium, provided the original author and source are credited 


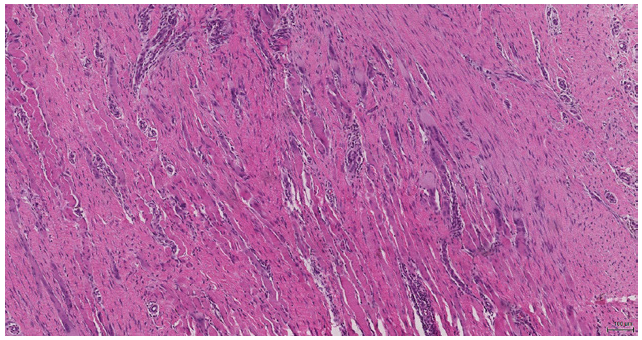

Figure 1: Spindled myofibroblastic cells arranged in fascicles, this is consistent with a diagnosis of a Desmoid tumour.

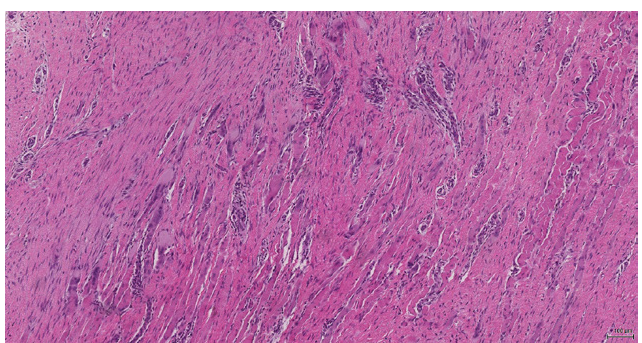

Figure 2: Histology showing an inked resection margin with myofibroblastic cells inflitrating into and through the skeletal muscle. There are also perivascular lymphoid infiltrates present. This I consistent with an abdominal wall desmoid tumour (fibromatosis) which has been incompletely excised.

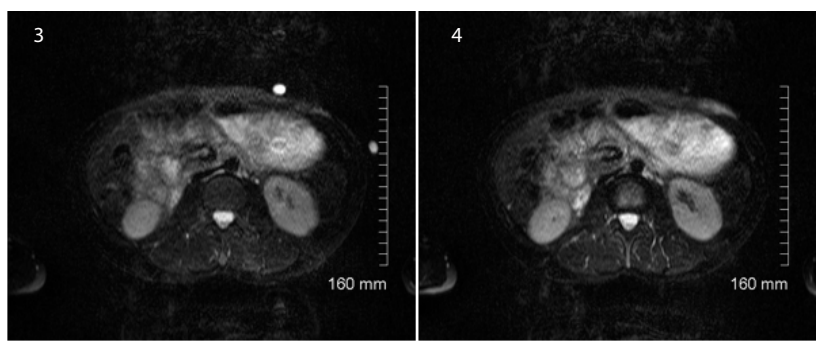

Figures 3 and 4: Altered signal in the subcutaneous tissues in the left uppe quadrant of the anterior abdomial wall measureing $6 \mathrm{~cm}$. This may represent residual desmoid tumour or recent surgical post-operative changes.

DF's. Ballo et al. reported that local spread was influenced negatively by increased tumour size and age over 30 years [10]. They also reported that there was no increased risk of local recurrence in those who had positive margins. Another study, reported that age, gender, surgical margin and adjuvant radiation therapy were not predictors of recurrence [11].

The need for negative margins when resecting a desmoid tumour is debated in the literature. Some studies reported that obtaining negative margins was important for local disease free survival $[8,12]$. However, another study concluded that there was no difference in local disease free survival in patients based on their margin status [13]. There is a high risk of local recurrence in patients with desmoid tumours in all of these studies.

\section{Case Report}

A 38 year old female presented to the General Surgical Outpatients Department, University Hospital Waterford with a painless, left hypochondrial mass. This mass was noted following the birth of her second child. The patient reported symptoms of night sweats, weight loss and pruritis of the skin overlying the mass. On examination, the mass was found to be firm, non-tender and fixed to the underlying abdominal wall. A past medical history of a breast fibroadenoma excision and LLETZ procedure was noted. There was no recent history of abdominal wall trauma or surgery. No relevant family history was noted.

Pre-operative abdominal wall ultrasound demonstrated a fusiform nodule within the left anterior abdominal transversalis oblique muscle, measuring $17 \mathrm{~mm} \times 7.4 \mathrm{~mm}$ and located inferior to the left costal cartilage. The lesion was reported to be solid with a focal hypervascular nidus. The advice given by the radiologist was for a surgical excision biopsy.

Surgical excision of the mass was performed under general anaesthetic. A skin ellipse was initially performed, followed by a muscle splitting procedure for removal of the soft tissue involved. Macroscopically, the mass was described as soft, firm, white and rubbery. A 3-layer abdominal wall closure was performed.

Histologically, the tumour was found to contain spindled myofibroblastic cells arranged in fascicles with perivascular lymphoid infiltrates. These myofibroblastic cells were found to be infiltrating through the skeletal muscle. The histological diagnosis was found to be an incompletely excised abdominal wall desmoid tumour. (Figures 1 and 2). The histopathological advice was for a further surgical resection of positive margins, given the high risk of recurrence of this tumour type.

A post-operative abdominal MRI was performed to evaluate for further tumour sites or extension and to allow for planning of surgical re-resection. The findings of the MRI were inconclusive, with altered signal in the subcutaneous tissues present in the left upper quadrant of the anterior abdominal wall of $6 \mathrm{~cm}$. This could represent residual desmoids tumour versus recent surgical changes or post contrast changes (Figures 3 and 4).

A further surgical resection was undertaken. The previous surgical scar was excised using a skin ellipse. An abdominal wall muscle margin resection was performed and the deep sheath was left intact. The circumferential fascia was released in order to get adequate wound approximation. A two layer fascia closure was performed. The defect was filled with Artiss tissue glue and the skin ellipse was refashioned.

The re-excision histology showed mainly benign skeletal muscle. There were residual areas of deep abdominal fibromatosis present adjacent to the previous surgical site. The lesion measured $25 \mathrm{~mm}$ in maximum dimension. Margins were all negative which were $0.7 \mathrm{~mm}$, $1 \mathrm{~mm}, 1.5 \mathrm{~mm}$ and $>5 \mathrm{~mm}$ in the superior, anterior, lateral and the remaining margins, respectively. A mesh was not placed during this surgical excision so as to allow for a further excision if margins were positive. An abdominal wall hernia repair may be required in the future [14].

\section{Discussion}

DF's are rare tumours which are benign but invade locally. There is a lack of treatment consensus within the literature. DF's more commonly occur in young females of child-bearing age. Therefore, deciding on the most appropriate treatment option should be done on a case by case basis. A watch and wait approach will not be suitable in patients which have an aggressively spreading desmoid tumour. Surgery can be a mutilating treatment option and radiation therapy can result in radiation related complications.

The treatment of DF's should be undertaken as part of 
Citation: Norton SM, McDermott A, Sehgal R, O'Donoghue G (2017) Invasion of the Anterior Abdominal Wall: A Case Report of a Desmoid Tumour. J Mol Biomark Diagn S2: 027. doi:10.4172/2155-9929.S2-027

a multidisciplinary team. If surgical resection is undertaken physiotherapy and rehabilitation is an important aspect of maximising functional status in order to prevent morbidities.

\section{Conflict of Interest}

There is no conflict of interest.

\section{References}

1. Agha RA, Fowler AJ, Saeta A, Barai I, Rajmohan S, et al. (2016) For the SCARE Group. The SCARE Statement: Consensus-based surgical case report guidelines. Int J Surg 34: 180-186.

2. Stout AP (1954) Juvenile fibromatoses. Cancer 7: 953-978.

3. Hosalkar HS, Torbert JT, Fox EJ, Delaney TF, Aboulafia AJ, et al. (2008) Musculoskeletal Desmoid tumours. J Am Acad Orthop Surg 16: 188-198.

4. Gurbuz AK, Giardiello FM, Petersen GM, Krush AJ, Offerhaus GJ, et al. (1994) Desmoid tumours in familial adenomatous polyposis. Gut 35: 377-381.

5. Lazar AJ, Tuvin D, Hajibashi S, Habeeb S, Bolshakov S, et al. (2008) Specific mutations in $\beta$-catenin gene (CTNNB1) correlate with local recurrence in sporadic desmoid tumors. Am J Pathol 173: 1518-1527.

6. Deyrup AT, Tretiakova M, Montag AG (2006) Estrogen receptor- $\beta$ expression in extraabdominal fibromatoses-an analysis of 40 cases. Cancer 106: 208-213.

7. Ghert M, Yao X, Corbett T, Gupta AA, Kandel RA, et al. (2014) Treatment and follow-up strategies in desmoid tumours: A practice guideline. Curr Oncol 21 642-649.

8. Spear MA, Jennings LC, Mankin HJ, Spiro IJ, Springfield DS, et al. (1998) Individualising management of aggressive fibromatoses. Int J Radiat Oncol Biol Phys 40: 637-645.

9. Guadagnolo BA, Zagars GK, Ballo MT (2008) Long-term outcomes for desmoid tumours treated with radiation therapy. Int J Radiat Oncol Biol Phys 71: 441-447.

10. Peng PD, Hyder O, Mavros MN, Turley R, GroeschI R, et al. (2012) Management and recurrence patterns of desmoid tumours: A multi-institutional analysis of 211 patients. Ann Surg Oncol 19: 4036-4042.

11. Karabulut S, Keskin S, Ekenel M, Basaran M, Agaoglu F, et al. (2013) The clinical effect of a positive surgical margin and adjuvant postoperative radiotherapy in the treatment of resectable desmoid tumors. Molecular and clinical oncology 1: 1061-1064

12. Ballo MT, Zagars GK, Pollack A, Pisters PW, Pollock RA (1999) Desmoid tumor: prognostic factors and outcome after surgery, radiation therapy, or combined surgery and radiation therapy. J Clin Oncol 17: 158-167.

13. Merchant NB, Lewis JJ, Woodruff JM, Leung DH, Brennan MF (1999) Extremity and trunk desmoid tumors: a multifactorial analysis of outcome. Cancer 86 : 2045-2052.

14. Briand S, Barbier O, Biau D, Bertrand-Vasseur A, Larousserie F, et al. (2014) Wait-and-See policy as a First-Line Management for Extra-Abdominal Desmoid Tumours. J Bone Joint Surg Am 96: 631-638.
This article was originally published in a special issue, Cancer Biomarkers handled by Editor(s). Dr. Sudhir Srivastava, Cancer Biomarkers Research Group, National Institute of Health, USA; Dr. Shou-Jiang Gao, The University of Texas Health Science Centre at San Antonio, USA; Dr. Kenneth Maiese, University of Medicine \& Dentistry of New Jersey, New Jersey Medical School, USA 